\title{
Desenvolvimento Sustentável no Brasil e o desafio da implementação da Política Nacional de Resíduos Sólidos
}

\author{
Sustainable Development in Brazil and the challenge of implementing the National Solid Waste \\ Policy
}

El desarrollo sostenible en Brasil y el desafío de implementar la Política Nacional de Residuos Sólidos

Rosana Santos de Almeida ORCID: https://orcid.org/0000-0001-8097-1074 Universidade Federal de Campina Grande, Brasil E-mail: rosana.santos@estudante.ufcg.edu.br

Agílio Tomaz Marques

ORCID: https://orcid.org/0000-0001-8364-5063 Universidade Federal de Campina Grande, Brasil E-mail: agiliotomaz@hotmail.com

Hugo Sarmento Gadelha ${ }^{1}$

ORCID: https://orcid.org/0000-0001-9414-0554 Universidad del Museo Social Argentino, Argentina E-mail: hugoscurso@uol.com.br

Hiran Mendes Castro Filho ${ }^{2}$

ORCID: https://orcid.org/0000-0002-1418-159X Universidad del Museo Social Argentino, Argentina E-mail: hirancastro@gmail.com

Adryele Gomes Maia

ORCID: https://orcid.org/0000-0002-7433-7138 Universidade Federal do Rio Grande do Norte, Brasil E-mail: adryelegm@gmail.com

Suzana Araújo dos Santos

ORCID: https://orcid.org/0000-0001-5955-9421

Universidade Federal de Campina Grande, Brasil

E-mail: suzana.santos2007@yahoo.com.br

Raquel Formiga de Medeiros ${ }^{3}$

ORCID: https://orcid.org/0000-0002-1198-5015 Universidad del Museo Social Argentino, Argentina E-mail: raquelfdm@hotmail.com

Matheus Matos Ferreira Silva

ORCID: https://orcid.org/0000-0002-3905-1951

Universidade Federal de Campina Grande, Brasil E-mail: matheusmatosfs@gmail.com

\begin{abstract}
Resumo
A presente pesquisa tem como objetivo analisar os desafios que surgiram através da implementação da Política Nacional dos Resíduos Sólidos (PNRS) à luz do princípio do desenvolvimento sustentável. Nos últimos anos, grandes impactos têm surgido no Brasil em decorrência do aumento do consumo de produtos gerado pelo capitalismo, e consequente o descarte de resíduos sólidos vêm agravando a poluição ambiental fazendo com que o governo e a sociedade se preocupem com a forma de descarte desses resíduos. A partir dessa preocupação foi criada a Lei 12.305/2010 que deu origem à PNRS onde o art. $6^{\circ}$ da referida lei prevê o desenvolvimento sustentável como um de seus princípios norteadores que se podem perceber por meio de diversas outras normas previstas como a gestão do ciclo dos materiais e a responsabilidade compartilhada dos poluidores. A pesquisa foi elaborada por meio da utilização do método de abordagem dedutivo, através da pesquisa bibliográfica, e documental. Através dos resultados encontrados foi possível constatar que muito embora a Lei 12.305/2010 tenha sido um passo importante na busca de
\end{abstract}

\footnotetext{
${ }^{1}$ Doutoranda pela Universidad del Museo Social Argentino.

${ }^{2}$ Doutorando pela Universidad del Museo Social Argentino.

${ }^{3}$ Doutoranda pela Universidad del Museo Social Argentino.
} 
Research, Society and Development, v. 11, n. 2, e39211225446, 2022

(CC BY 4.0) | ISSN 2525-3409 | DOI: http://dx.doi.org/10.33448/rsd-v11i2.25446

soluções para o descarte de resíduos sólidos nas últimas décadas, ainda não se mostra suficiente para solucionar a questão, tendo em vista que os municípios não estão sequer colocando em prática as determinações previstas do referido dispositivo legal, sendo indispensável a ação integrada entre o governo, estados e os municípios, bem como da contribuição das empresas privadas e da população em geral.

Palavras-chave: Consumo; Desenvolvimento sustentável; Política Nacional de Resíduos Sólidos.

\begin{abstract}
This research aims to analyze the challenges that arose through the implementation of the National Solid Waste Policy (PNRS) in light of the principle of sustainable development. In recent years, large impacts have arisen in Brazil as a result of the increased consumption of products generated by capitalism, and consequently the disposal of solid waste has aggravated environmental pollution, causing the government and society to worry about how to dispose of these waste. From this concern, Law 12,305/2010 was created, which gave rise to the PNRS where art. 6 of the aforementioned law provides for sustainable development as one of its guiding principles, which can be seen through several other standards, such as the management of the material cycle and the shared responsibility of polluters. The research was elaborated through the use of the deductive approach method, through bibliographic and documentary research. Through the results found, it was possible to verify that, although Law 12,305/2010 has been an important step in the search for solutions for the disposal of solid waste in recent decades, it is still not enough to solve the issue, considering that the municipalities they are not even putting into practice the determinations provided for in the aforementioned legal provision, and integrated action between the government, states and municipalities, as well as the contribution of private companies and the population in general, is essential.
\end{abstract}

Keywords: Consumption; Sustainable development; National Solid Waste Policy.

\title{
Resumen
}

Esta investigación tiene como objetivo analizar los desafíos que surgieron a través de la implementación de la Política Nacional de Residuos Sólidos (PNRS) a la luz del principio de desarrollo sostenible. En los últimos años, han surgido grandes impactos en Brasil como resultado del mayor consumo de productos generados por el capitalismo y, en consecuencia, la disposición de desechos sólidos ha agravado la contaminación ambiental, provocando que el gobierno y la sociedad se preocupen por cómo eliminar estos desechos. A partir de esta inquietud, se creó la Ley 12.305 / 2010, que dio origen al PNRS donde el art. 6 de la citada ley prevé el desarrollo sostenible como uno de sus principios rectores, que puede verse a través de varios otros estándares, como la gestión del ciclo material y la responsabilidad compartida de los contaminadores. La investigación se elaboró mediante el uso del método del enfoque deductivo, mediante la investigación bibliográfica y documental. A través de los resultados encontrados se pudo constatar que, si bien la Ley 12.305 / 2010 ha sido un paso importante en la búsqueda de soluciones para la disposición de residuos sólidos en las últimas décadas, aún no alcanza para resolver el tema, considerando que el municipios ni siquiera están poniendo en práctica las determinaciones previstas en la referida disposición legal, y es fundamental la acción integrada entre el gobierno, estados y municipios, así como el aporte de las empresas privadas y de la población en general.

Palabras clave: Consumo; Desenvolvimiento sustentable; Política Nacional de Residuos Sólidos.

\section{Introdução}

A geração excessiva de lixo, aliada a falta de destino adequado dos resíduos sólidos, têm sido foco de várias discussões e pesquisas que vem buscando alguns tipos de soluções para tentar organizar esse descarte, e neste sentido, o Brasil vem procurando desenvolver algumas alternativas, e para isso aprovou um marco regulatório para gestão de resíduos sólidos (Pozeti \& Caldas, 2019).

Na busca dessas alternativas, foi instituída a partir da criação da Lei no 12.305/2010 a Política Nacional de Resíduos Sólidos que te em suas diretrizes a incumbência o intuito de incentivar a promoção de uma gestão integrada ao gerenciamento de resíduos sólidos, atribuindo aos gestores públicos a responsabilidade para o desenvolvimento de projetos para promoção da gestão integrada e o gerenciamento ambiental adequado dos resíduos sólidos no país.

Diante da preocupação da produção excessiva e da destinação final ambientalmente segura desses resíduos sólidos, têm acontecido diversas discussões e pesquisas que buscam alternativas para tentar solucionar essa preocupação, e neste sentido, o Brasil vem procurando desenvolver algumas alternativas, para isso aprovou um marco regulatório para gestão de resíduos (Pozeti \& Caldas 2019). 
Research, Society and Development, v. 11, n. 2, e39211225446, 2022

(CC BY 4.0) | ISSN 2525-3409 | DOI: http://dx.doi.org/10.33448/rsd-v11i2.25446

Entre as saídas para enfrentar a problemática dos resíduos sólidos seria a racionalidade, pois indica a necessidade de que a sociedade possa pensar e escolher o melhor caminho, considerando a importância do assunto, uma das saídas desse processo seria a utilização da política dos 3Rs (reduzir, reutilizar e reciclar). Para essa alternativa, alguns autores denominam de princípios e outros como política, visando contribuir como um instrumento eficaz para solução dos problemas do lixo e melhorar o bem-estar das pessoas (Andrade, 2014).

Em face a essa problemática, essa pesquisa buscou contribuir para que seja feita uma reflexão sobre como a gestão dos resíduos sólidos tem se dado no contexto da sustentabilidade brasileira. Tendo como objetivo geral analisar o desafio da implementação da Política Nacional de Resíduos Sólidos, frente ao desenvolvimento sustentável.

Adotou-se como metodologia para a realização desse estudo o método de abordagem dedutivo partindo da análise de como se dá o desenvolvimento sustentável no Brasil frente ao desafio da implementação da Política Nacional de Resíduos Sólidos, sendo feita também uma abordagem qualitativa, por meio de pesquisa exploratória e descritiva, realizada na forma de pesquisa bibliográfica, desenvolvida a partir de materiais publicados em livros, artigos, dissertações e teses e pesquisa documental através de análise de leis constitucionais e infraconstitucionais.

Para uma melhor compreensão esse artigo está dividido em três partes: no primeiro capitulo foi feito um contexto histórico sobre o desenvolvimento sustentável no Brasil, o segundo capítulo trata da aplicabilidade da Lei n 12.305/2010 que trata dos resíduos sólidos, por fim o terceiro capítulo discorre sobre a Política Nacional de Resíduos Sólidos à luz do sistema jurídico brasileiro.

Na conclusão apresentaremos um posicionamento crítico, que irá discutir a relação à teórica que abordará sobre o descarte de resíduos sólidos e sua relação com a sustentabilidade, considerando a relação que emerge da necessidade de novos posicionamentos dos governos e sociedade brasileira.

\section{Aplicabilidade da Lei $\mathrm{n}^{\circ} \mathbf{1 2 . 3 0 5 / 2 0 1 0}$}

Um dos maiores desafios já enfrentados pela política e gestão do desenvolvimento sustentável brasileiro tem acontecido principalmente em consequência do gerenciamento inadequado dos resíduos sólidos que tem gerado danos a longo prazo ao meio ambiente e à saúde da população, o que consequentemente tem contribuindo para perdas econômicas do país.

Diante dessa preocupação da produção excessiva e da destinação final ambientalmente segura desses resíduos sólidos, vem acontecendo muitas discussões e pesquisas que buscam alternativas para tentar solucionar esse problema, e neste sentido, o Brasil vem procurando desenvolver algumas alternativas, e para isso aprovou o Marco Regulatório para Gestão de Resíduos (Pozeti \& Caldas 2019).

Foi a partir da aprovação, a Lei nº 12.305/2010 que se instituiu a Política Nacional de Resíduos Sólidos (PNRS) que busca em suas diretrizes incentivar e promover a gestão integrada e o gerenciamento desses resíduos, seja qual for sua natureza, imputando aos gestores públicos a responsabilidade para o desenvolvimento de projetos para promoção da gestão integrada e ao gerenciamento ambientalmente adequado dos resíduos sólidos no país.

Inicialmente a criação da PNRS, tinha como meta $100 \%$ do país com a cobertura de soluções sustentáveis de tratamento até a data de 02/08/2014. Porém infelizmente, até o ano de 2014, mais de 60\% dos municípios no país continuavam com o manejo inadequado, de acordo com os dados Associação Brasileira de Empresas de Limpeza Pública e Resíduos Especiais (ABRELPE) e, o restante, ainda contava com o uso indiscriminado da terra para a destinação dos resíduos (Neto, 2019).

A PNRS institui nos seus artigos art. $\left(1^{\circ}, \S 1^{\circ}\right.$, art. $3^{\circ}$, inciso IX, art. 25) que os destinatários das disposições legais: São as pessoas físicas ou jurídicas, de direito público ou privado, responsáveis, direta ou indiretamente, pela geração de 
resíduos sólidos por meio de suas atividades, nelas incluído o consumo, e as que desenvolvam ações relacionadas à gestão integrada ou ao gerenciamento de resíduos sólidos.

Nesse sentido, percebe-se que a lei prevê no seu Art. 25. Que o poder público, o setor empresarial e a coletividade são responsáveis pela efetividade das ações voltadas para assegurar a observância da Política Nacional de Resíduos Sólidos e das diretrizes e demais determinações estabelecidas nesta Lei e em seu regulamento.

Essa problemática que envolve os resíduos sólidos vem sendo discutida a anos e ganhou mais impulso a partir da Rio92 e se intensificou durante toda a década de 1990, quando o consumo surgiu como questão de política ambiental relacionada às propostas de sustentabilidade. Como consequência a comissão para o Desenvolvimento Sustentável, criada dentro da Rio92 deu início a um programa de pesquisas para examinar, os desafios aos impactos ambientais dos padrões de consumo (Portilho, 2010).

O intuito principal da implantação do manejo adequado de resíduos sólidos, é representar a necessidade que sociedade tem em cumprir requisitos básicos como: gerar menos resíduos e o desenvolvimento de sistema efetivo de gestão de forma sustentável que seja economicamente viável.

Dessa forma, para que ocorra uma política de gestão ambiental do lixo é preciso que seja tomada a adoção de sistemas de Gestão Integral de Resíduos GIR, combinação de fluxos de geração com métodos de coleta, tratamento e disposição final com benefícios ambientais otimizados sob o ponto de vista econômico e social. Para que isso aconteça esse sistema deve ser sustentável em termos ambientais, econômicos e social, com orientação para o mercado, flexível e operado em escala regional (Barakat, 2009).

Para que haja uma destinação racional dos resíduos, sejam eles urbanos ou industriais, é preciso que seja justificada, primeiramente, pela necessidade de evitar a sua simples deposição e contaminação do ambiente e, em segunda instância, pela possibilidade de se auferir renda a partir de sua reutilização. Por sua importância, o processo de descarte de resíduos sólidos, vem sendo discutido e realizado de forma gerencial, de modo a fomentar nas sociedades um caráter de integração ao ciclo econômico e de inserção social (Gameiro, 2011).

Mesmo com a implementação da (PNRS) ainda necessário caminhar para que a implementação da gestão dos resíduos que garanta a eliminação de impactos negativos no ambiente e na saúde da população. E a partir da aprovação da Política Nacional de Resíduos Sólidos (PNRS) de 2010, alguns municípios tem trabalhado para erradicação dos lixões e implantação de coleta seletiva, embora esbarrem na limitação da destinação em aterros sanitários ou incineração.

Entre as saídas para enfrentar a problemática do lixo seria a racionalidade, pois indica a necessidade de que a sociedade possa pensar e escolher o melhor caminho, considerando a importância do assunto, uma das saídas desse processo seria a utilização da política dos 3Rs (reduzir, reutilizar e reciclar). Para essa alternativa, alguns autores denominam de princípios e outros como política, visando contribuir como um instrumento eficaz para solução dos problemas do lixo e melhorar o bem-estar das pessoas (Andrade, 2014).

\section{A Política Nacional de Resíduos Sólidos a Luz do Sistema Jurídico Brasileiro}

De acordo com a lei brasileira, a responsabilidade civil em matéria ambiental é um tema que sempre se renova e evolui, adquirindo importância cada vez maior nos estudos de direito ambiental, de fato, por mais que se prestigie a tutela estritamente preventiva do meio ambiente, diante da realidade por todos conhecida, de que as agressões ao meio ambiente, uma vez consumadas, são de difícil, custosa e incerta reparação, não há como negar que, frequentemente, os mecanismos preventivos se mostram limitados e insuficientes à preservação e à conservação da qualidade ambiental (Dias, 2009). 
Uma dessas penalidades de danos civil ao meio ambiente diz respeito ao princípio do poluidor pagador que implica na responsabilidade dos fabricantes diante de externalidades negativas que podem ser geradas durante do processo de produção. Segundo as externalidades podem ser positivas, também chamada de economia externa, ou negativa, conhecida por deseconomia externa (Dias 2009).

Essa, inclusive, é a orientação firmada pelo Superior Tribunal de Justiça, em expressivo acórdão da lavra do ministro Herman Benjamin:

[...] 3. A jurisprudência do STJ está firmada no sentido de que, nas demandas ambientais, por força dos princípios do poluidor-pagador e da reparação in integrum, admite-se a condenação, simultânea e cumulativa, em obrigação de fazer, não fazer e indenizar. Assim, na interpretação do art. $3^{\circ}$ da Lei 7.347/1985, a conjunção 'ou' opera com valor aditivo, não introduz alternativa excludente. Precedentes da Primeira e Segunda Turmas do STJ. 4. A recusa de aplicação, ou aplicação truncada, pelo juiz, dos princípios do poluidor-pagador e da reparação in integrum arrisca projetar, moral e socialmente, a nociva impressão de que o ilícito ambiental compensa, daí a resposta administrativa e judicial não passar de aceitável e gerenciável 'risco ou custo normal do negócio'. Saem debilitados, assim, o caráter dissuasório, a força pedagógica e o objetivo profilático da responsabilidade civil ambiental (= prevenção geral e especial), verdadeiro estímulo para que outros, inspirados no exemplo de impunidade de fato, mesmo que não de direito, do degradador premiado, imitem ou repitam seu comportamento deletério.

Não se trata, evidentemente, de permitir a poluição mediante singelo pagamento. a noção do princípio do poluidor pagador apresenta duas vertentes: a) um caráter preventivo, pela procura na evitação do dano ambiental; e b) reparação do dano provocado (Fiorillo, 2009).

Como podemos ver, vigora em nosso sistema jurídico o princípio da reparação integral ou in integrum do dano ambiental, muito parecido com o princípio do poluidor-pagador, ao determinar a responsabilização por todos os efeitos decorrentes da conduta lesiva, incluindo, entre outros aspectos, o prejuízo suportado pela sociedade, até que haja completa e absoluta recuperação in natura do bem lesado.

Na mesma linha desse julgado estão os arts. 225, 170, inciso VI, e 186, II, da Constituição Federal, e o art. $4^{\circ}$ da Lei n. 6.938/1981 as quais dispõem que a Política Nacional do Meio Ambiente se norteará pelos princípios do poluidor-pagador, do usuário pagador e da reparação in integrum, concretizados por meio da obrigação de recuperar o dano ambiental; indenizar os prejuízos sofridos pelas vítimas e pela biota afetada; e pagar pelos serviços ambientais retirados da Natureza.

\section{Resultados e Discussão}

A Política Nacional dos Resíduos Sólidos apresenta uma visão sistêmica e preventiva da disposição dos resíduos na medida em que trata de todo o ciclo de vida dos produtos deixando clara a necessidade de se reduzir a produção de resíduos sólidos, e que, conforme o disposto no art. $5^{\circ}$ da Lei 12.305/2010, integra a Política Nacional do Meio Ambiente e articula se com a Política Nacional de Educação Ambiental e com a Política Federal de Saneamento Básico (Matias \& Menezes 2018).

A implementação da PNRS, foi de extrema importância no senário brasileiro ao mesmo tempo que ela trata da destinação dos resíduos sólidos aliado ao desenvolvimento sustentável dessa forma essa lei vai de encontro ao art. $5^{\circ}$ da Carta Magna que demostra uma preocupação ambiental para preservação do meio ambiente para gerações futuras.

Nesse sentido o art. 51 da Lei 12.305/2010 estabelece de forma expressa que a ação ou omissão das pessoas físicas ou jurídicas que não observarem o disposto na referida lei irá gerar o dever de reparar os danos causados, independentemente da existência desculpa, sem prejuízo das demais sanções cabíveis. 
Research, Society and Development, v. 11, n. 2, e39211225446, 2022

(CC BY 4.0) | ISSN 2525-3409 | DOI: http://dx.doi.org/10.33448/rsd-v11i2.25446

Isso implica dizer que as pessoas geradoras desses resíduos sólidos devem ter conhecimento das consequências geradas pelo descarte e destinação inadequada desses resíduos sólidos e não podem alegar por hipótese alguma o desconhecimento da referida lei sob pena de reparação do dano civil que tenha sido causado.

Dessa forma, a previsão da responsabilidade compartilhada das pessoas físicas ou jurídicas que derem causa a danos ambientais pela Política Nacional de Resíduos Sólidos demonstra que o caráter sistêmico e integrador da referida lei está presente tanto no que concerne ao ciclo dos materiais quanto à responsabilidade por danos ambientais, independente do momento do ciclo em que ocorra o dano e da existência de culpa (Matias \& Menezes 2018).

De forma que as decisões que envolvem o gerenciamento de resíduos sólidos urbanos são fundamentalmente decisões que tratam sobre a saúde pública e requerem, a integração de todos os sujeitos que estejam envolvidos nesse ciclo, seja entre políticas econômicas, sociais e ambientais.

Outra discussão, diz respeito ao complexo desafio para as grandes cidades na gestão de resíduos sólidos neste início de século pode ser enfrentado pela formulação de políticas públicas que objetivem eliminar os riscos à saúde e ao ambiente, que colaborem na mitigação das mudanças climáticas relacionadas à ação humana e, ao mesmo tempo, garantam a inclusão social efetiva de parcelas significativas da população (Golveia, 2012).

Assim, nessa perspectiva, caminharemos rumo a um desenvolvimento sustentável mais saudável, em um viés socialmente justo, ambientalmente sustentável, sanitariamente correto e economicamente solidária e sustentável para que a sociedade e empresários e órgãos governamentais trabalhem em conjunto para a promoção da preservação ambiental.

\section{Considerações Finais}

Através da pesquisa foi possível constatar que a falta de gerenciamento adequado dos resíduos sólidos causa danos irreparáveis e permanentes ao meio ambiente o que influencia na qualidade de vida de pessoas e outras espécies. Dessa forma devem ser tomadas algumas medidas que podem atenuar os danos ambientais, sendo uma delas o gerenciamento integrado dos resíduos sólidos.

Nesse contexto, é de extrema importância a percepção desta problemática, por parte de toda a sociedade, empresários e governantes envolvidos, tendo como viés a responsabilidade social que ajudará o futuro do planeta. Através dessa discussão também se faz necessário incentivar, construir e implementar ações que viabilizem essa transformação. Como por exemplo a política dos 3R's que vem mostrando resultados satisfatórios ao longo da sua aplicação.

Portanto, é perceptível que a sustentabilidade exerce função essencial dessa visão de transformação e adaptação, entretanto, para que seja viável depende principalmente da consciência, precaução, responsabilidade e cooperação, razão crucial de um sinérgico avanço do conhecimento sobre gestão de resíduos sólidos na relação com a sustentabilidade.

Diante disso, é possível dizer que a gestão de resíduos sólidos deve ser integrada e compartilhada, mas também sustentável, para isso, é necessário que ocorra uma gestão econômica e ambientalmente sustentável, assim a sociedade atual e futura estará sendo contemplada, bem como alavancar o processo de comprometimento dos responsáveis que geram os resíduos.

\section{Referências}

Associação Brasileira de Empresas de Limpeza Pública e Resíduos Especiais (Abrelpe) (2010). Panorama de Resíduos Sólidos no Brasil- São Paulo: https://abrelpe.org.br/

Andrade, J. B. L., (2012). Indicadores de Sustentabilidade Aplicáveis à Gestão e Política Públicas para os Resíduos Sólidos Industriais: uma construção com foco no Polo Industrial de Manaus: EDUA, 2014.

Barakat, Munir. (2009). Sustentabilidade Ambiental e Gestão de Resíduos Sólidos Urbanos: Uma análise do modelo de gestão da CIA Norte. Universidade Federal do Paraná. [dissertação]. Curitiba: Programa de Pós-graduação em Desenvolvimento Econômico, Universidade Federal do Paraná. 
Research, Society and Development, v. 11, n. 2, e39211225446, 2022

(CC BY 4.0) | ISSN 2525-3409 | DOI: http://dx.doi.org/10.33448/rsd-v11i2.25446

Brasil. (2010). Lei 12.305. Institui a Política Nacional de Resíduos Sólidos. Brasília-DF, https://fld.com.br/catadores/pdf/politica_residuos_solidos.pdf.

Dias, G. M. (2009). Cidade Sustentável. Natal. https://www.e-inscricao.com/Natalsustentavel/seminario

Fiorillo, C. A. P., (2009). Curso de Direito Ambiental Brasileiro. (10a ed.), Saraiva.

Gameiro, A. H. et al. (2011). Logística Ambiental de Resíduos Sólidos. Editora Atlas S.A.

Gouveia, N. (2012). Resíduos sólidos urbanos: impactos socioambientais e perspectiva de manejo sustentável com inclusão social. Ciência \& Saúde Coletiva 17(6). https://doi.org/10.1590/S1413-81232012000600014.

Mantovaneli, Jr. O., (2013). Gestão sustentável, habitus e ação: princípios esquecidos pela agenda do desenvolvimento. ed. EDIFURB. ISBN: 9788571141971.

Martins Jr, J., (2015). Como escrever trabalhos de conclusão de curso: instruções para planejar e montar, desenvolver, concluir, redigir e apresentar trabalhos monográficos e artigos. (9a ed.), Vozes.

Matias, N. J. L., \& Menezes, L. T. (2018). Analise da política nacional de resíduos sólidos a luz do paradigma do desenvolvimento sustentável. Revista do programa de pós graduação em direito UFC. 38(2).

Neto, O. P. R. (2019). Modelagem do impacto socioeconômico do tratamento integrado de resíduos sólidos na economia brasileira. In: Instituto Venturi para estudos ambientais. Porto Alegre. http://www.institutoventuri.org.br/ojs/index.php/firs/issue/view/Anais-FIRS-2019.

Portilho, F. (2010). Sustentabilidade ambiental, consumo e cidadania. (2a ed.), Cortez.

Pozzeti, V. C. \& Caldas, J. N. (2019). O descarte de resíduos sólidos no âmago da sustentabilidade. Revista de Direito Econômico e Socioambiental, Curitiba, 10(1), 183-205, 10.7213/rev.dir.econ.soc.v10i1.24021.

Silva, J. A. da. (2009). Direito Ambiental Constitucional. (7a ed.), Malheiros.

STJ (2011). $2^{\mathrm{a}}$ T. - REsp n. 1145083/MG - j. 27.09.2011, rel. Min. Herman Benjamin. 